\title{
ARTICLE
}

\section{Development of a Current-Readout Type Neutron Monitor for Burst Neutron Fields}

\author{
Kazuhiko IIJIMA*, Toshiya SANAMI, Masayuki HAGIWARA, Kiwamu SAITO, Shin-ichi SASAKI \\ ${ }^{1}$ High Energy Accelerator Research Organization, 1-1 Oho, Tsukuba, Ibaraki 305-0801, Japan
}

\begin{abstract}
We report on the development of a current-readout type neutron monitor for the measurement of the dose rate of burst neutrons generated by beam loss in an accelerator that occurs within a short time span. This neutron monitor consists of a commercially available neutron proportional counter, a $6.5-\mathrm{cm}$ thick polyethylene moderator, and a newly developed charge-integration circuit. The monitor was tested under mono-energetic neutron standard fields (En $=8 \mathrm{keV}$ to $15 \mathrm{MeV}$ ) and was compared with a conventional pulse-readout type monitor. The energy response of the current-readout monitor is identical to that of the pulse-readout monitor. The current-readout monitor is able to measure neutron doses up to $4.5 \mathrm{mSv} / \mathrm{h}$ without count loss, whereas the pulse-readout monitor fails due to count loss.
\end{abstract}

\section{KEYWORDS: Neutron Radiation Monitor, Burst Field, ${ }^{3} \mathrm{He}$ proportional counter, Current readout}

\section{Introduction}

In recent years, beam intensity and beam energy at accelerators have increased rapidly due to the demands placed by applications such as neutron science and nuclear transmutation. Accelerators generate intense radiation fields because of beam loss at target and beam dumps. For the purpose of radiation control, shielding walls and radiation monitors are required around the accelerator. In particular, the radiation field around a proton synchrotron is generated during a very short time period (these fields are hereinafter called burst fields) because the beam-extraction period after acceleration is less than several hundred $\mu$ s for a typical synchrotron.

Figure 1 shows an example of the difference between peak dose rates under direct-current (DC) and pulse-mode operation. As shown in this figure, the peak dose rate under pulse-mode operation is 20,000 times higher than under DC-mode operation even for the same integrated dose. In this example, even for an integrated dose of $1 \mu \mathrm{Sv}$ over 1 hour, the radiation monitor should

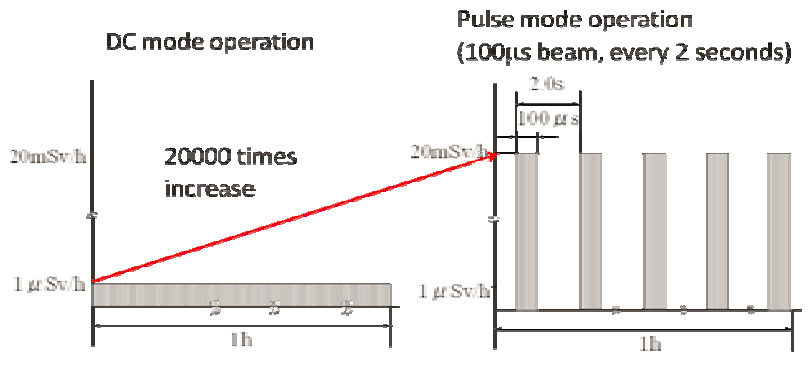

Integrated dose $=$ I $15 \mathrm{v}$ for 1 hour

Fig. 1 Comparison between peak dose rates under DC and pulse-mode operation. The peak dose rate under pulse-mode operation is 20,000 times higher than under DC-mode operation for same integrated dose.

*Corresponding Author, E-mail:kazuhiko.iijima@kek.jp measure a peak dose rate of $20 \mathrm{mSv} / \mathrm{h}$ if the accelerator operates with a $100-\mu$ s wide beam and a repetition rate of $0.5 \mathrm{~s}^{-1}$. The operation generates a burst radiation field around the accelerator. Because of count loss, the dose rate of the burst field might be measured correctly using a commercially available radiation monitor with pulse readout.

A current-readout ionization chamber is one candidate to measure radiation without count loss. Ionization chambers have been commercially available for photons. Thus, a current-readout monitor for neutrons is required for accurate monitoring of radiation from the accelerators.

In this study, a current-readout type neutron monitor was developed. This neutron monitor consists of a commercially available neutron proportional counter, a $6.5-\mathrm{cm}$ thick polyethylene moderator, and a newly developed charge integration circuit. With this monitor, neutron sensitivity, energy response, acceptable range of neutron intensity, and sensitivity to gamma rays were obtained experimentally.

\section{Current-readout neutron monitor}

\section{Methodology for current readout}

Figure 2 shows a block diagram of a newly developed current-readout type pre-amplifier circuit. The circuit was originally developed for a readout signal from an ionization chamber. The circuit was modified to operate a commercially available neutron proportional counter under ionization-chamber mode by supplying positive high voltage to the outer tube instead of the center wire. Because of this inverse high-voltage configuration, electron multiplication around the central wire is restrained. The induced signals on the wire due to electron and ion drift are accumulated in a condenser (C1). The condenser is automatically discharged using a relay (SW1) after reaching a predefined voltage. The procedure is repeated cyclically. 


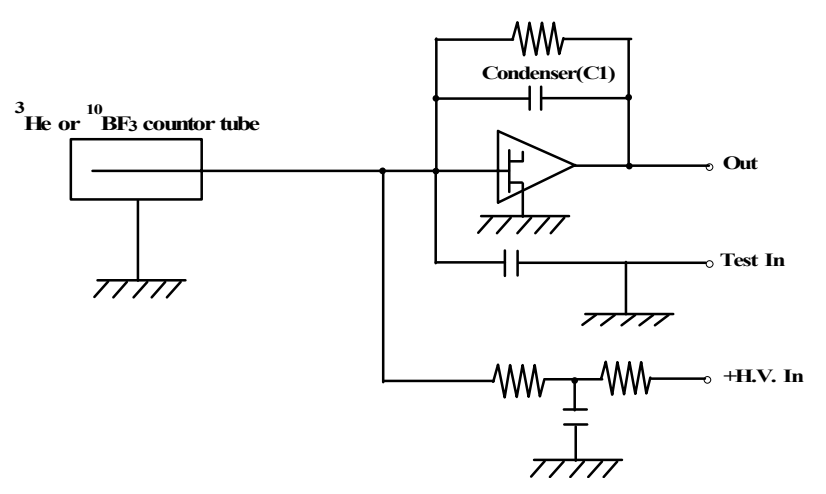

Fig. 2 Block diagram of the current-readout type pre-amplifier circuit.

Figure 3 shows a schematic of the output signal from the circuit under low- (above) and high-dose rate (below) conditions. In this example, the voltage to reset $\mathrm{C} 1$ is set to $1 \mathrm{~V}$. The period between two adjacent resets is varied depending on the dose rate. As shown in Fig. 3, the slope gradient due to voltage increase under high-dose rate is larger than that under low-dose rate. The sensitivity of the circuit is controlled by adjusting the interval time between two adjacent resets. The gradient is converted to a logic pulse by using a digital differential circuit unit connected to the output of the charge-integration circuit. Thus, the detector output can be read as a series of pulses instead of using an ammeter. The pulse rate is adjusted to $1 \mathrm{cps}$ under background conditions (no neutrons) mainly because of leaking current.

The current-readout circuit can read out induced current in a commercially available neutron proportional
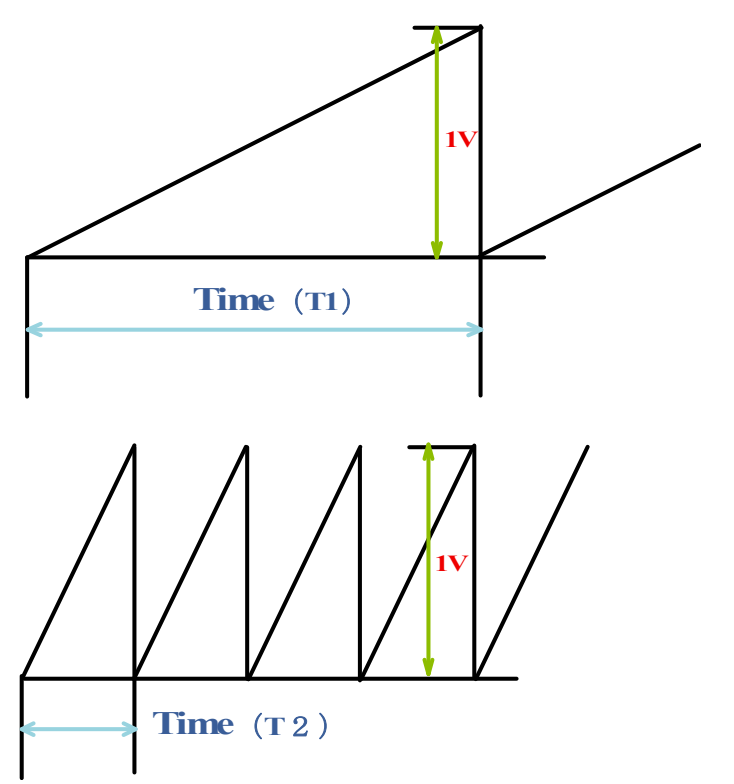

Fig. 3 Schematic diagram of the output signal from the circuit under low- (above) and high-dose rate (below) conditions. The period between two adjacent resets is varied depending of the dose rate. counter. However, the threshold of the measurable dose rate could be higher than for a conventional pulse-readout circuit, because the current from the proportional counter would be very low. The measurable-dose threshold should be checked to confirm the applicability of the current-readout method. In a later section, the capabilities of the current-readout method are examined experimentally using neutron and gamma-ray sources, and the results are compared with a conventional pulse-readout circuit.

\section{Construction of the neutron monitor}

To construct a neutron monitor based on the current-readout method, the current-readout circuit is mounted on the case of a conventional pre-amplifier unit that could be connected directory to the 1- or 2-inch diameter proportional counter. The proportional counter is available commercially (Reuter-Stokes Co. Ltd.). The proportional counter contains ${ }^{3} \mathrm{He}$ counting gas at $9.3 \mathrm{~atm}$ or ${ }^{10} \mathrm{BF}_{3}$ at 1 atm. To operate the counter in ionization-chamber mode using the circuit shown in Fig. 2, $500 \mathrm{~V}$ is supplied to the outer counter wall. The applied high voltage was determined based on the relationship between the signal output rate and the high voltage by changing it from 300 to $1000 \mathrm{~V}$ to avoid electron-ion recombination.

Figure 4 shows a schematic view of the neutron monitor. The counter is covered with a $6.5-\mathrm{cm}$ thick polyethylene moderator to enhance sensitivity to fast neutrons. The thickness of the moderator is determined by considering the shape of the response function and the sensitivity. The response could easily be improved by

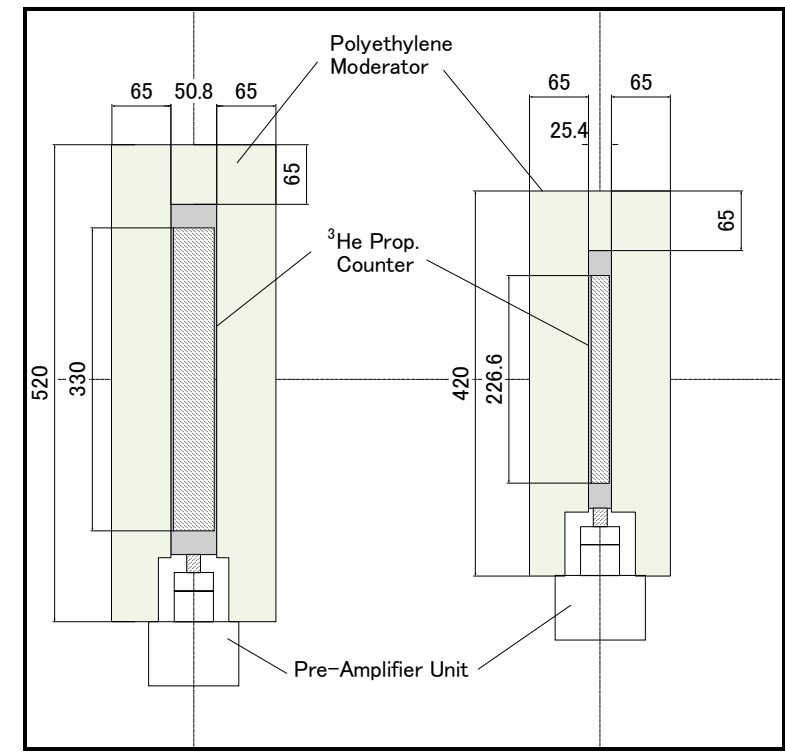

Fig. 4 Schematic view of the neutron-monitor setup. The neutron proportional counter with 2-inch (left) or 1-inch (right) diameter is inserted in a $6.5-\mathrm{cm}$ thick polyethylene moderator. The counter is connected directory to the pre-amplifier unit that contains the circuit for current readout or pulse readout. 
replacing the moderator to that containing tungsten ${ }^{1)}$.

\section{Performance test}

The neutron counter with the current-readout circuit was tested for its (1) calibration factor, (2) energy response, (3) dose-rate linearity, and (4) gamma-ray sensitivity.

\section{Calibration factor}

The signal of the current-readout circuit should be calibrated. The calibration factor of the current readout method was determined relative to the dose rate of the pulse-readout neutron monitor that was already calibrated. An Am-Be neutron source was used for this calibration. The ratio of counts between two different readout monitors was obtained for the source placed on the moderator. Table 1 summarizes the results of calibration constants for different types of detectors and readouts with counter specifications. As shown in this table, the ratios (pulse readout / current readout) are $\sim 500$ and $\sim 240$ for ${ }^{3} \mathrm{He}$ and ${ }^{10} \mathrm{BF}_{3}$ gas, respectively. The difference between these ratios is due to the difference in gas pressures, $\mathrm{Q}$ values of reaction, and $\mathrm{W}$ values of the counting gas. The gas pressure of both ${ }^{3} \mathrm{He}$ counters is $68948 \mathrm{~Pa}(10 \mathrm{psi})$. The gas pressure of $1-$ and 2-inch ${ }^{10} \mathrm{BF}_{3}$ counters are $400 \mathrm{~mm} \mathrm{Hg}$ and $700 \mathrm{~mm} \mathrm{Hg}$, respectively. The $\mathrm{Q}$ values of neutron-detection reactions for ${ }^{3} \mathrm{He}$ and ${ }^{10} \mathrm{BF}_{3}$ are $0.764 \mathrm{MeV}$ and $2.79 \mathrm{MeV}$, respectively.

\section{Energy response}

The energy response of the neutron counters was measured using the neutron reference field (FRS) at the Japan Atomic Energy Agency $(\text { JAEA })^{2,3,4)}$. Figure $\mathbf{5}$ shows the experimental setup. The neutron monitors with current and pulse readout were placed $200 \mathrm{~cm}$ from the neutron production target. Mono-energetic neutrons at $8 \mathrm{keV}, 144$ $\mathrm{keV}, 250 \mathrm{keV}, 565 \mathrm{keV}, 5.0 \mathrm{MeV}$, and $14.8 \mathrm{MeV}$ were chosen as energy points to obtain responses. Neutron fluences at the position were determined from the counts of long counters placed at $110^{\circ}$ and that were already calibrated. The contribution from room-scattered neutrons was subtracted using the data obtained with a shadow cone.

Figure 6 shows the energy response obtained from the experiment (symbols) and Monte-Carlo simulation calculated by MCNPX code ${ }^{5)}($ lines $)$ for the 1 -inch

Table 1 Results of calibration factors for different types of

detectors and readouts.

\begin{tabular}{|c|c|c|c|}
\hline \multirow{2}{*}{$\begin{array}{c}\text { Proportional } \\
\text { counter types }\end{array}$} & \multicolumn{2}{|c|}{$\begin{array}{c}\text { Calibration constant } \\
{[(\mu \mathrm{Sv} / \mathrm{h}) / \mathrm{cps}]}\end{array}$} & \multirow{2}{*}{$\begin{array}{c}\text { Ratio } \\
\text { Current/Pulse }\end{array}$} \\
\cline { 2 - 3 } & $\begin{array}{c}\text { Current } \\
\text { readout }\end{array}$ & Pulse readout & \\
\hline 1 inch ${ }^{3} \mathrm{He}$ & 34.5 & 0.07 & 493 \\
\hline 2 inch ${ }^{3} \mathrm{He}$ & 13.8 & 0.0273 & 505 \\
\hline 1 inch ${ }^{10} \mathrm{BF}_{3}$ & 72.5 & 0.32 & 226 \\
\hline 2 inch ${ }^{10} \mathrm{BF}_{3}$ & 11.6 & 0.053 & 219 \\
\hline
\end{tabular}

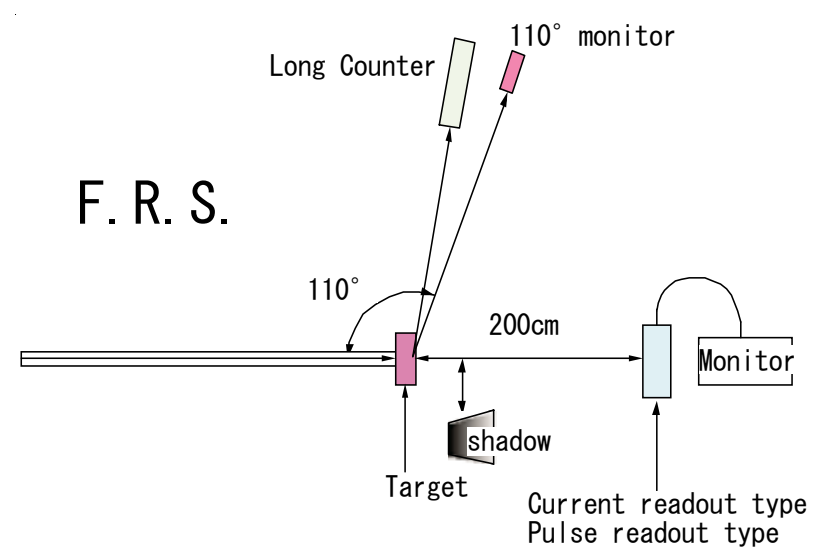

Fig. 5 Experimental setup to obtain energy response of neutron monitor with current and pulse readout.

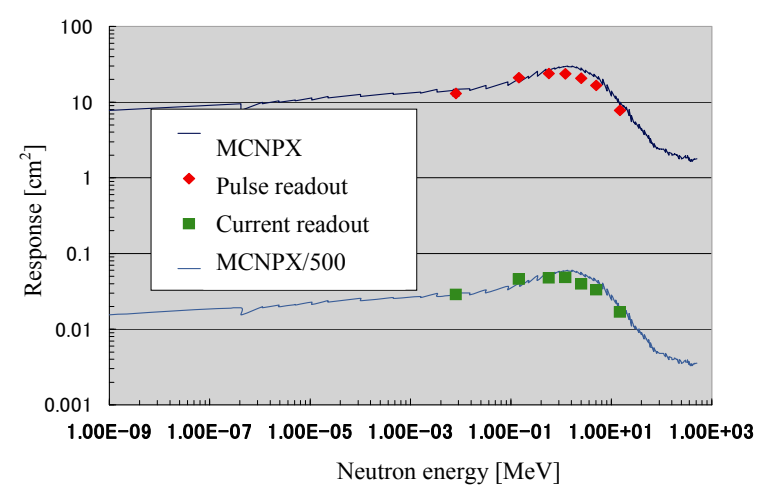

Fig. 6 Energy response obtained from the experiments (symbols) and Monte-Carlo simulation calculated by MCNPX code (lines).

diameter ${ }^{3} \mathrm{He}$ counter with moderator. The current- and pulse-readout neutron monitors show identical energy dependence. Only the magnitude of the current readout is $\sim 500$ times smaller than the pulse readout.

\section{Dose-rate linearity}

Dose-rate linearity was examined using a $5-\mathrm{MeV}$ mono-energetic neutron beam at FRS. Figure 7 shows the result obtained by neutron monitors with current- and pulse-readout circuits as a function of count rate of the relative monitor (long counter). The contributions from room scattering were subtracted. The measured dose rates were obtained as an average over $300 \mathrm{~s}$.

From Fig. 7, the data of neutron monitor with pulse-readout circuit shows $10 \%$ count losses at $300 \mu \mathrm{Sv} / \mathrm{h}$ and $50 \%$ at $1700 \mu \mathrm{Sv} / \mathrm{h}$. However, the data with current readout shows no count loss up to $1700 \mu \mathrm{Sv} / \mathrm{h}$. These results clearly show the advantage of the current-readout circuit with regard to count loss.

The lower limit of the measurable dose rate is found to be $4 \mu \mathrm{Sv} / \mathrm{h}$ under this condition. This rate is slightly high for the purpose of environmental radiation monitoring. The lower limit could be improved by changing the sensitivity of 


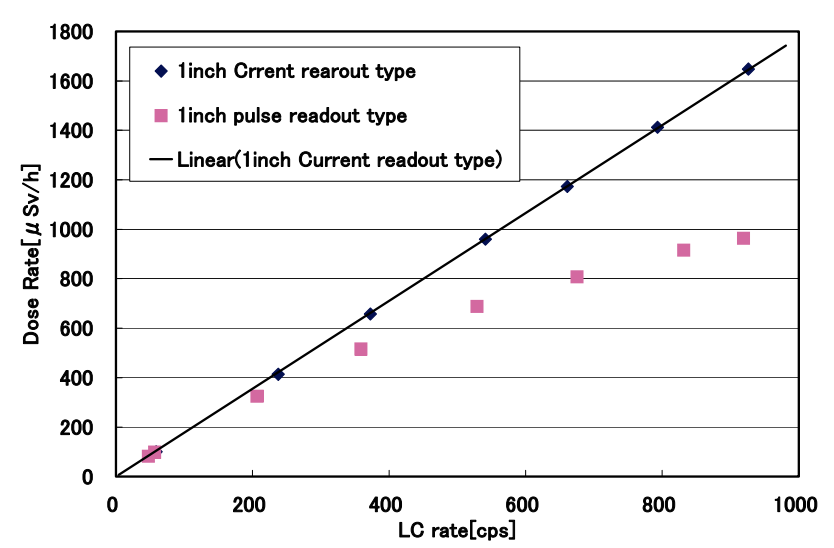

Fig. 7 Linearity of dose rate measured by neutron monitors with current- and pulse-readout circuits as a function of count rate of the relative monitor (long counter).

the current-readout circuit and the averaging time of the measurement.

\section{Gamma-ray sensitivity}

The current-readout method cannot distinguish a gamma-ray signal from a neutron signal because both signals are immediately integrated in the circuit. Thus, the output signal of the current readout circuit contains a certain contribution from gamma rays if they exist in the field. The sensitivity to gamma rays was experimentally estimated using a radioactive source emitting gamma rays. Figure 8 shows the experimental layout to obtain the gamma-ray sensitivity. The sources ${ }^{137} \mathrm{Cs}(3.7 \mathrm{GBq})$ and ${ }^{60} \mathrm{Co}(1.85$ GBq) were used. The result is that $4 \%$ and $8 \%$ of the actual gamma-ray dose is recorded by the current-readout circuit with 1 -and 2 -inch diameter ${ }^{3} \mathrm{He}$ proportional

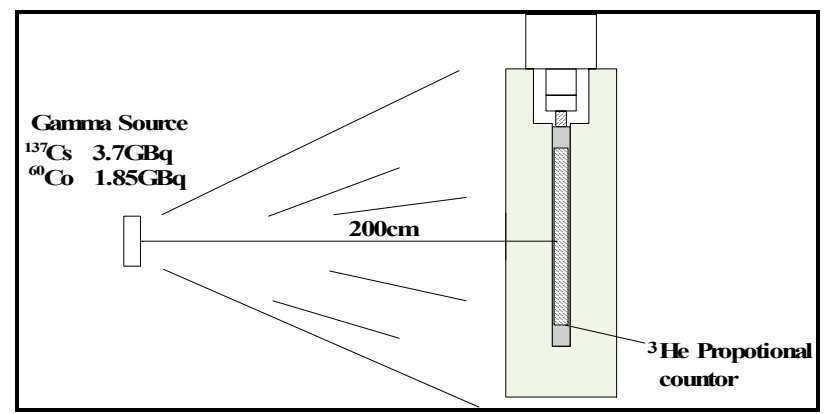

Fig.8 Experimental layout for gamma-ray sensitivity test.

counters, respectively.

\section{Summary}

A neutron monitor with a current-readout circuit is developed to avoid underestimating the dose of burst neutrons because of count loss. The current-readout circuit can be connected to the commercially available ${ }^{10} \mathrm{BF}_{3}$ and ${ }^{3} \mathrm{He}$ proportional counters to operate them under ionization-chamber mode. The combination of circuit, proportional counter, and polyethylene moderator, which serves as a neutron-radiation monitor, is tested experimentally for its (1) calibration factor, (2) energy response, (3) dose-rate linearity, and (4) gamma-ray sensitivity. From the results, we reach the following conclusions:

- The energy response of the neutron monitor with current readout is equal to that found with pulse readout.

- Excellent linearity is found in comparison with the conventional pulse-readout neutron monitor.

- Confirmed dynamic range of the neutron monitor is from 4 $\mu \mathrm{Sv} / \mathrm{h}$ to $5 \mathrm{mSv} / \mathrm{h}$.

- $4 \%$ and $8 \%$ of actual gamma-ray doses are recorded by a neutron monitoring system consisting of 1- and 2-inch ${ }^{3} \mathrm{He}$ proportional counters, respectively, with the current readout circuit.

The neutron monitor has been tested in burst fields around proton and electron accelerators in high-energy accelerator research organization ${ }^{6}$.

\section{Acknowledgment}

The authors wish to thank the operators of FRS for their cooperation with the neutron experiment. This neutron experiment was performed under the Common-Use Facility Program of JAEA.

\section{References}

1) M. Hagiwara, T. Sanami, T. Michikawa, S. Sasaki," Development of Neutron Monitors with Wide Range Dose Response form Thermal to Gev" , KEK Proceedings 2007-12 Radiation Detectors and Their Uses(2007)193

2) Y. Tanimura, M. Yoshizawa, J. Saegusa, K. Fujii, S. Shimizu, M. Yoshida, Y. Shibata, A. Uritani, K. Kudo, Radiat. Prot. Dosim., 110,85 (2004).

3) Y. Tanimura, J. Saegusa, Y. Shikaze, M. Tsutsumi, S. Shimizu, M. Yoshizawa, Radiat. Prot. Dosim., 126, 8 (2007).

4) Y. Shikaze, Y. Tanimura, J. Saegusa, M. Tsutsumi, S. Shimizu, M. Yoshizawa, Y. Yamaguchi, J. Nucl. Sci. Technol., Suppl. 5, 209 (2008).

5) Gregg W. McKinney et al., MCNPX 2.6.X Features (2006-2007), LA-UR-07-2053, M\&C/SNA 2007 Conference Workshop, Monterey, CA, April 15-19, 2007.

6) K. Iijima, T. Sanami, M. Hagiwara, K. Saito, H. Nakamura, S. Sasaki, "Development and field test of realtime radiation monitor for intense pulsed neutron field", KEK Proceedings 2008-14 Radiation Detectors and Their Uses(2008)160 